\title{
A simplified system for the effective expression and delivery of functional mature microRNAs in mammalian cells
}

\author{
Jiaming Fan ${ }^{1,2} \cdot$ Yixiao Feng ${ }^{2,3} \cdot$ Ruyi Zhang ${ }^{2,4} \cdot$ Wenwen Zhang ${ }^{2,3} \cdot$ Yi Shu $^{2,3} \cdot$ Zongyue Zeng $^{1,2}$ - Shifeng Huang ${ }^{2,3}$. \\ Linghuan Zhang ${ }^{2,3} \cdot$ Bo Huang ${ }^{1,2,5} \cdot \mathrm{Di} \mathrm{Wu}^{2} \cdot$ Bo Zhang ${ }^{2,6} \cdot \mathrm{Xi}$ Wang ${ }^{1,2} \cdot$ Yan Lei ${ }^{2,3} \cdot$ Zhenyu Ye $^{2,7} \cdot$ Ling Zhao $^{2,3}$.

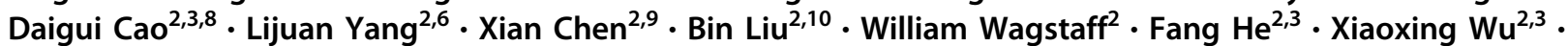 \\ Jing Zhang ${ }^{2,3} \cdot$ Jennifer Moriatis Wolf ${ }^{2} \cdot$ Michael J. Lee $^{2} \cdot$ Rex C. Haydon ${ }^{2} \cdot$ Hue H. Luu ${ }^{2} \cdot$ Ailong Huang $^{1}$. \\ Tong-Chuan $\mathrm{He}^{2} \cdot$ Shujuan $\mathrm{Yan}^{2,11}$
}

Received: 30 March 2019 / Revised: 21 May 2019 / Accepted: 1 June 2019 / Published online: 20 June 2019

(c) The Author(s) 2019. This article is published with open access

\begin{abstract}
MicroRNAs (miRNAs) are $~ 22$ nucleotide noncoding RNAs that are involved in virtually all aspects of cellular process as their deregulations are associated with many pathological conditions. Mature miRNAs (mMIRs) are generated through a series of tightly-regulated nuclear and cytoplasmic processing events of the transcribed primary, precursor and mMIRs. Effective manipulations of miRNA expression enable us to gain insights into miRNA functions and to explore potential therapeutic applications. Currently, overexpression of miRNAs is achieved by using chemically-synthesized miRNA mimics, or shRNA-like stem-loop vectors to express primary or precursor miRNAs, which are limited by low transfection efficacy or rate-limiting miRNA processing. To overcome rate-limiting miRNA processing, we developed a novel strategy to express mMIRs which are driven by converging U6/H1 dual promoters. As a proof-of-concept study, we constructed mMIR expression vectors for hsa-miR-223 and hsa-Let-7a-1, and demonstrated that the expressed mMIRs effectively silenced target gene expression, specifically suppressed miRNA reporter activity, and significantly affected cell proliferation, similar to respective primary and precursor miRNAs. Furthermore, these mMIR expression vectors can be easily converted into retroviral and adenoviral vectors. Collectively, our simplified mMIR expression system should be a valuable tool to study miRNA functions and/or to deliver miRNA-based therapeutics.
\end{abstract}

\section{Introduction}

The completion of the Human Genome Project and the advent of high-throughput deep sequencing technologies have revealed that, while eukaryotic genomes are pervasively transcribed, $<2 \%$ of the human genome is transcribed into protein-coding mRNA, which leaves most of the transcribed human genome noncoding RNAs (ncRNAs) [16]. Based on their sizes, ncRNAs are divided into two

Supplementary information The online version of this article (https:// doi.org/10.1038/s41417-019-0113-y) contains supplementary material, which is available to authorized users.

Tong-Chuan He

tche@uchicago.edu

Shujuan Yan

shujyan@qq.com

Extended author information available on the last page of the article groups: small ncRNAs ( $<200 \mathrm{nt})$ and long ncRNAs or IncRNAs (>200 nt) [7, 8]. While the biological functions of ncRNAs remain to be fully understood, increasing evidence suggests that ncRNAs play critical regulatory roles in numerous cellular processes [5, 7-11]. Nonetheless, small ncRNAs, such as microRNAs and small interfering RNAs (siRNAs), have received much attention since their discoveries.

MicroRNAs (miRNAs or miRs) are small noncoding RNAs of $\sim 22$ nucleotides (nt) in length, which induce gene silencing by guiding Argonaut (AGO) proteins to completely or partially complementary binding sites at the $3^{\prime}$ untranslated region (UTR) of target mRNAs [12-18]. The first miRNA was discovered in 1993 in Caenorhabditis elegans as a short RNA produced by the lin- 4 gene, which post-transcriptionally represses the lin-14 mRNA [19-21]. Such small regulatory RNAs were later found abundantly presented in diverse animal phyla and were subsequently named microRNAs [13]. Currently, the miRNA repository 
miRBase lists 1917 precursor miRNAs (pMIRs) and 2654 mature miRNAs (mMIRs) for humans [22], and it has been estimated that $>60 \%$ of human protein-coding genes harbor predicted miRNA target sites [23].

The short single-stranded miRNAs are initially transcribed as longer primary transcripts (or termed pri-miRNAs), containing a 60-120 nt RNA hairpin in which one of the two strands includes the mMIR[13]. The hairpincontaining pri-miRNAs are successively cleaved by two RNase III enzymes, Drosha in the nucleus and Dicer in the cytoplasm, to yield $\sim 70 \mathrm{nt}$ pMIRs and $22 \mathrm{nt}$ mMIRs, respectively [13]. The pMIRs are transported to the cytoplasm via Exportin-5 and further processed by Dicer to produce a short, partially double-stranded RNA, in which one strand is the mMIR. mMIRs modulate gene expression posttranscriptionally by imperfectly binding target mRNAs in association with the AGO-containing multi-protein RNAinduced silencing complex [13]. AGOs are a large family of proteins that use single-stranded small nucleic acids as guides to complementary sequences in RNA or DNA targeted for silencing [13, 24]. The miRNA-loaded AGO forms the targeting module of the miRNA-induced silencing complex, leading to translation repression and/or degradation of targeted mRNAs $[13,25]$. Nonetheless, recent evidence has revealed that miRNA processing steps may follow canonical processing routes, and/or many noncanonical miRNA biogenesis pathways, which crosstalk with other cellular pathways [17].

It is well established that miRNAs are involved in virtually every cellular process and are essential for development, cell differentiation, and homeostasis [13]. In fact, deregulation of miRNA function has been associated with human diseases [12, 26], particularly in cancers [13, 27, 28], as miRNAs can function as both oncogenes (or oncomiRs) [29] and tumor suppressors [30], although miRNA expression is generally downregulated in most cancers $[13,27,28,31]$. Thus, it is highly desirable to effectively manipulate the exogenous miRNA expression in order to gain insights into their biological functions, and in some cases, to explore their potential therapeutic applications. Downregulation or inhibition of miRNA functions can be usually accomplished by the use of anti-miRs, antagomiRs, AMOs (anti-miRNA antisense oligonucleotides), miRNA sponges, miRNA decoys, or circularized anti-miRs, most of which are usually based on antisense molecules to bind and sequester miRNAs from their natural targets [18, 32-34]. On the other hand, upregulation or overexpression of miRNAs can be usually accomplished by the use of chemically synthesized miRNA mimics, or shRNA-like or intronic miRNA expression vectors to express the primary miRNAs (priMIRs) or pMIRs [35-37]. However, the efficacy of miRNA mimics is transient in nature and limited by transfection efficiency. The commonly used intronic miRNA expression strategy will rely on the endogenous miRNA processing efficiency and may cause cytotoxicity due to oversaturation of the RNAi machinery $[37,38]$. Thus, there is an unmet need to develop fully optimized miRNA-expressing vectors for the efficient expression of miRNAs in cultured cells and animals.

In order to overcome the rate-limiting siRNA/miRNA processing machinery, here we developed a novel and simplified strategy to express mMIRs by exploiting the converging $\mathrm{U} 6 / \mathrm{H} 1$ dual promoter-driven expression of miRNAs. We successfully used the converging U6/H1 dual promoter-driven system to express siRNAs [39, 40]. However, the asymmetric nature or imperfect complementarity of the $5 \mathrm{p}$-miR and 3p-miR sequences of a given miRNA requires a different design. We overcame this challenge by inserting the transcription stop signals (a string of TTTTTAAAAA) between the 5p-miR (in sense direction) and 3p-miR (in antisense direction) sequences to terminate the transcription of $5 \mathrm{p}-\mathrm{miR}$ and $3 \mathrm{p}-\mathrm{miR}$, respectively. As positive controls, we also constructed the U6-driven expression of pMIRs and the conventional priMIR expression systems.

As a proof-of-concept study, we constructed the pMIR and mMIR expression vectors for the commonly-studied hsa-miR-223 and hsa-let-7a-1. We demonstrated that the mMIRs effectively inhibited target gene expression, specifically suppressed target gene $3^{\prime}$-UTR-derived reporter activity, and effectively affected cell proliferation in a fashion similar to that of the respective priMIR and pMIRs expression systems in human cell lines. Furthermore, our mMIR expression vector was constructed on the bases of retroviral transfer and adenoviral shuttle vectors. Thus, the final mMIR expression constructs can be easily converted into recombinant retrovirus for stable expression or recombinant adenovirus for effective transient expression in vitro and in vivo. Collectively, our results demonstrate that the simplified mMIR expression system is user-friendly and reproducibly effective, which should be a valuable resource for studying miRNA functions and/or exploring potential applications of miRNA-based therapeutics.

\section{Materials and methods}

\section{Cell culture and chemicals}

HEK-293 and human colon cancer cell line HCT116 were obtained from the American Type Culture Collection (Manassas, VA, USA). The cells were maintained in the DMEM contained $10 \%$ fetal bovine serum, $100 \mu \mathrm{g}$ streptomycin and 100 units of penicillin at $37^{\circ} \mathrm{C}$ in $5 \% \mathrm{CO}_{2}$ as previously reported [41-44]. Unless indicated otherwise, all chemicals were purchased from Sigma-Aldrich (St. Louis, MO, USA) or Thermo Fisher Scientific (Waltham, MA, USA). 


\section{Construction of the three types of expression vectors for hsa-miR-223 (MIR223) and hsa-let-7a-1 (MIRLET7A1)}

As illustrated in Fig. 1, the conventional priMIR expression vector was constructed on our home-made adenoviral shuttle vector pAdTrace-TOX [45-47], which contains the CMV promoter-driven expression cassette and SV40 Pa. Genomic DNA fragments containing primary hsa-miR-223 (MIR223) and hsa-let-7a-1 (MIRLET7A1) were PCR amplified from HEK-293 genomic DNA and subsequently cloned into the pAdTrace-OK vector, resulting in priMIR223 and priMIRLET7A1 expression vectors, respectively.

To express the precursor miRs hsa-miR-223 (pMIR223) and hsa-let-7a-1 (pMIRLET7A1), we first engineered the retroviral pSEB-pmiR and adenoviral shuttle pAdTracepmiR vectors on the base of our previously characterized pSOS system [39], through the Gibson Assembly system (New England Biolabs, or NEB, Ipswich, MA) as reported [40] (Supplemental Fig. 1a, b). These vectors contain a U6 promoter followed by MluI and HindIII cloning sites. Subsequently, we cloned oligo cassettes containing the pmiR sequences for hsa-miR-223 and hsa-let-7a-1, followed by the TTTTT transcription termination signal, into the MluI and Hind $I I I$ cloning sites, resulting in pMIR223 and PMIRLET7A1.

For expressing mMIR's miR-5p and miR-3p simultaneously in one vector, we engineered the retroviral pSEB$\mathrm{miR}$ and adenoviral shuttle pAdTrace-miR vectors on the base of the pSOS system [39] through the Gibson Assembly system (NEB) [40] (Supplemental Fig. 2a, b). In these vectors, the expression of mature miR-5p and miR-3p is driven by the convergent $\mathrm{U} 6$ and $\mathrm{H} 1$ promoters in opposing directions, respectively, with respective transcriptional termination signals (TTTTT or UUUUU) inserted between the mature miRs. Accordingly, oligo cassettes containing the mature miR-5p and miR-3p sequences of hsa-miR-223 (mMIR223) and hsa-let-7a-1 (mMIRLET7A1) were cloned into the SalI and Hind III sites of the pAdTrace-miR and pSEB-miR vectors, resulting in mMIR223 and mMIRLET7A1 constructs, respectively. All cloning junctions and oligo cloningderived vectors were verified by DNA sequencing. Detailed information about the reported constructs and/or the reported vectors is available upon request.

\section{Cell transfection}

For the reported studies, freshly seeded subconfluent cells were transfected by using the linear polyethylenimine (PEI)based Transporter $5^{\mathrm{TM}}$ Transfection Reagent (Polysciences, Inc., Warrington, PA) according to the manufacturer's
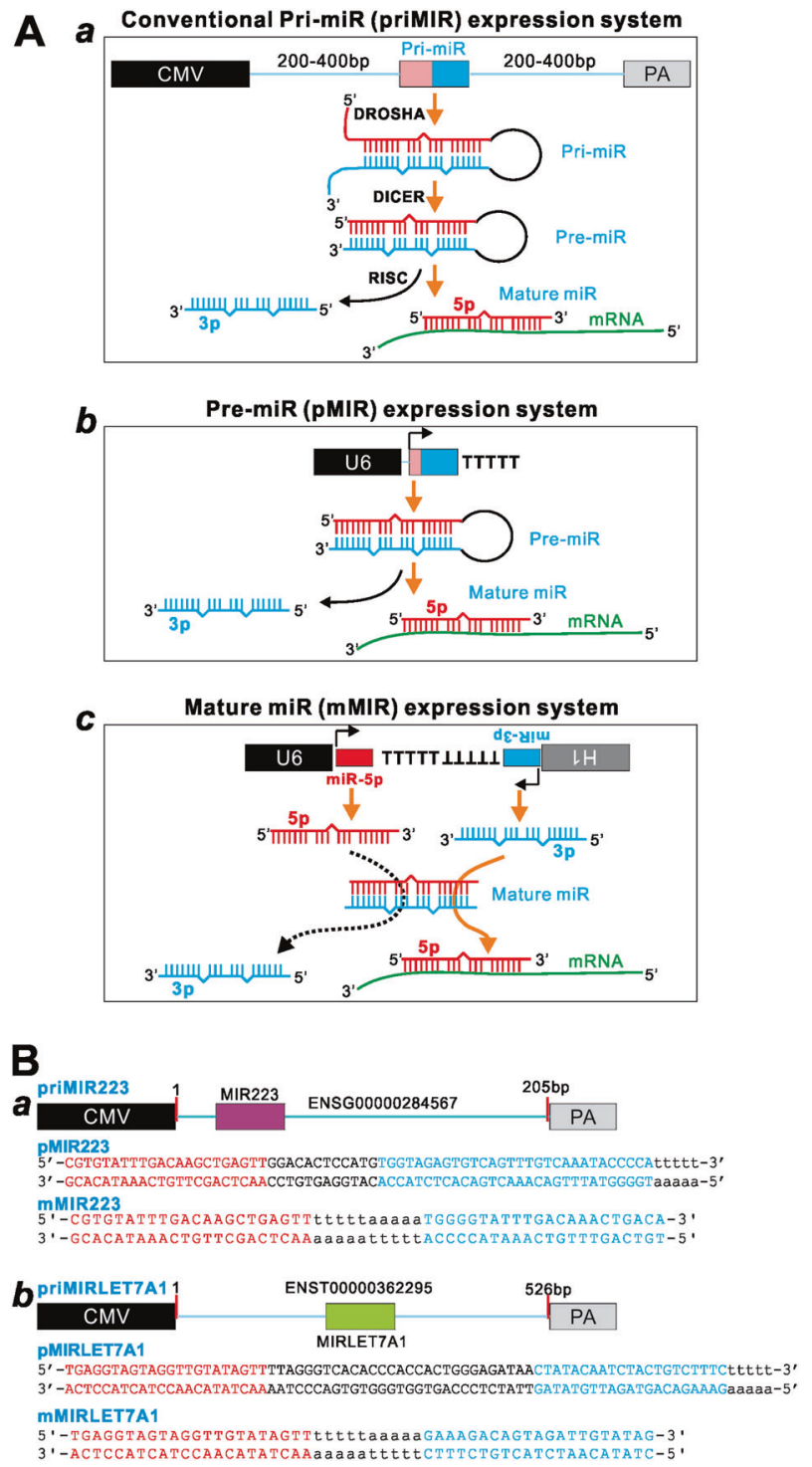

Fig. 1 Schematic representation of the three miRNA expression systems compared in this study. a Schematic representation of the three expression systems and possible modes of their processing and actions. The conventional primary miR (priMIR) expression system consists of cloning 200-500 bp genomic fragment surrounding the miR transcript unit under the CMV promoter $(a)$. The precursor miR (pMIR) expression system consists of the U6-driven expression of pre-miR sequence followed by a string of UUUUU (or TTTTT in the diagram) as the transcriptional termination signal $(b)$. The mature miR (mMIR) expression system is composed of the convergent $\mathrm{U} 6$ and $\mathrm{H} 1$ promoter-driven the expression of mature miR-5p and miR-3p in opposing directions, respectively, with the respective transcriptional termination signal (TTTTT or UUUUU) separating the U6 and H1 expression units. b Two representative miRs selected for the proof-of-principal study. The schematic genomic fragments and detailed miR sequences are shown for the tested three expression constructs of hsa-miR-223 (MIR223) (a) and hsamiR-let-7a-1 (MIRLET7A1) (b). miR, miRNA, or microRNA; CMV, cytomegalovirus promoter; U6, human U6 promoter; H1, human H1 promoter; PA, polyadenylation signal for terminating transcription

instructions. At the indicated time points, the transfected cells were collected for various assays described below. 


\section{Total RNA isolation and touchdown quantitative real-time PCR (TqPCR)}

At the endpoint, the transfected cells were subjected to total RNA isolation by using NucleoZOL Reagent (Takara Bio USA, Mountain View, CA) according to the manufacturer's introduction. For qPCR analysis of mRNA transcripts, total RNA was used for reverse transcription with the hexamer and M-MuLV (NEB). The cDNA products were diluted as templates for qPCR. The qPCR primers were designed by Primer3 Plus program [48]. For assessing the miR expression levels mediated by the three expression systems, the reverse transcription reactions were carried out by using miR-specific reverse primers that were complementary with the $3^{\prime}$-end six nucleotides of mature $\mathrm{miR}-5 \mathrm{p}$ and/or miR-3p, preceded with a 44-nt artificial stem-loop sequence. SYBR green-based quantitative real-time PCR analysis was performed by following our previously optimized TqPCR protocol [49]. The qPCR reactions were done in triplicate. All expression values were normalized to the reference gene $G A P D H$ expression by using the $2^{-\Delta \Delta C t}$ method [50-53]. The sequences of the qPCR primers are listed in Supplemental Table 1.

\section{Construction of miR-223 and let-7a-1 Gaussia luciferase (GLuc) reporters}

The GLuc reporters for miR-223 and let-7a-1 were constructed on the base of our recently characterized reporter vector pNRGLuc, which express Gaussia luciferase (GLuc) with a multiple cloning linker at the $3^{\prime}$-end of GLuc coding region [54, 55]. As human ARRBI and LIN28B are well established targets for miR-223 and let-7a-1, which contain miR-223 and let-7a-1-binding sites at their $3^{\prime}$-UTRs, respectively, we PCR amplified the $3^{\prime}$-untranslated sequences of human ARRB1 (or BUTR) and LIN28B, and subcolned them into pNRGLuc vector, resulting in pNRGLuc-BUTR (or GLuc-BUTR) and pNRGLucLIN28B (or GLuc-LIN28B), respectively. For the control reporters, mutations were introduced to the miR-binding sites and yielded pNRGLuc-BUTR Mut (or GLuc-BUTR Mut) and pNRGLuc-LIN28B Mut (or GLuc-LIN28B Mut). All PCR amplified sequences were verified by DNA sequencing.

\section{Gaussia luciferase (GLuc) reporter assay}

The GLuc reporter assays were carried out as described [56-58]. Briefly, exponentially growing HEK-293 and HCT116 cells were seeded in 12-well cell culture plates and coinfected with different combinations of miR expression vectors and/or the GLuc reporter plasmids. At $72 \mathrm{~h}$ after transfection, the cell culture media were subjected to GLuc activity assays using the BioLux GLuc Assay Kit (NEB). Each assay condition was done in triplicate.

\section{Cell proliferation WST-1 assay}

The WST-1 cell proliferation assay was performed as described [59-62]. Experimentally, exponentially growing cells were seeded in $60 \mathrm{~mm}$ cell culture dishes and transfected with miR expression plasmids or the control vector. At $16 \mathrm{~h}$ after transfection, the transfected cells were replated into 96-well cell culture plates at $30 \%$ confluence. Unseeded wells were added with culture medium and utilized as background controls. At the indicated time points, the premixed WST-1 (Takara Bio USA) was added to each well and incubated at $37^{\circ} \mathrm{C}$ for $2 \mathrm{~h}$. The plates were subjected to a microtiter plate reader to obtain absorbance reading at $450 \mathrm{~nm}$. Each assay condition was done in triplicate.

\section{Crystal violet staining assay}

The crystal violet cell viability assay was carried out as described [41, 63-65]. Briefly, subconfluent cells seeded in $35 \mathrm{~mm}$ cell culture dishes and transfected with miR expression plasmids or vector control. At 3 days after transfection, the cells were subjected to crystal violet staining. Macrographic images of the stained dished were recorded. Each condition was done in triplicate.

\section{Cell cycle analysis}

Cell cycle analysis was conducted as previously described [57, 66, 67]. Exponentially growing HEK-293 and HCT116 cells were seeded in $60 \mathrm{~mm}$ dishes and transfected with let-7a-1 expression plasmids or vector control. At $48 \mathrm{~h}$ post transfection, cells were collected and stained with the Magic Solution (containing 0.05\% NP-40, 4\% formaldehyde, $0.01 \mu \mathrm{g} / \mathrm{ml}$ Hoechst 33258 in PBS) for $30 \mathrm{~min}$ [68]. The stained cells were subjected to flow cytometry analysis using BD FACSCalibur-HTS. The flow cytometry data were quantitatively analyzed by FlowJo software. Each assay condition was performed in triplicate.

\section{Statistical analysis}

The quantitative assays were performed in triplicate and/or repeated in three independent batches. Data were expressed as mean \pm standard deviation. The one-way analysis of variance was used to analyze statistical significance. A value of $p<0.05$ was considered statistically significant. 


\section{Results}

\section{The design of a novel mature miRNA expression system}

Towards simplifying the exogenous expression of miRNAs, we designed two systems to express pMIRs and mMIRs, and then compared their functionality with the conventional miRNA expression system, in which the priMIR expression is accomplished by using a Pol II promoter (e.g., CMV) to drive a $200-500$ bp genomic DNA fragment containing miRNA transcript unit (Fig. 1a, a). To express pMIRs, we constructed the pMIR expression system, in which human U6 promoter is used to drive the expression of pre-miR sequence followed by a string of five UUUUU (or TTTTT in the diagram) as the transcriptional termination signal (Fig. 1a, b). For the expression of mMIRs, we explored the possibility to use a converging U6/H1 dual promoter-driven system, which has been successfully used to express siRNAs [39, 40]. However, we had to overcome the asymmetric nature or imperfect complementarity of the $5 \mathrm{p}-\mathrm{miR}$ and $3 \mathrm{p}$-miR sequences of a given miRNA by inserting the transcription stop signals (a string of TTTTTAAAAA) between the $5 p-m i R$ (in sense direction) and 3p-miR (in antisense direction) sequences to terminate the transcription of $5 \mathrm{p}$ $\mathrm{miR}$ and $3 \mathrm{p}-\mathrm{miR}$, respectively. Thus, in this converging dual-promoter system, the mMIR expression is achieved by using the convergent $\mathrm{U} 6$ and $\mathrm{H} 1$ promoters to drive the expression of mature miR-5p and miR-3p in opposing directions, respectively, with the respective transcriptional termination signal (TTTTT or UUUUU) separating the U6 and $\mathrm{H} 1$ expression units (Fig. 1a, c). Both pMIR and mMIR expression systems have been engineered in the adenoviral shuttle vector and retroviral transfer vector so they can easily be used to generate recombinant adenoviruses or retroviruses (Supplementary Figs. 1 and 2).

To evaluate the functionality of mMIR expression system in comparison to the conventional pri-miRNA and pMIR expression systems, we carried out a proof-ofconcept study and focused on two well-studied miRNAs, hsa-miR-223 and hsa-let7a-1. Briefly, we constructed the pri-miR-223 expression vector by cloning the PCR amplified 200 bp human genomic DNA fragment containing miR-223 under the control of a CMV promoter, resulting in priMIR223. Similarly, the pMIR223 and mMIR223 were constructed to express pMIRs and mature miR-223, respectively (Fig. 1b,a). Using the same set of vectors, we constructed expression vectors for primary let7a-1 (priMIRLET7A1), precursor let-7a-1 (pMIRLET7A1), and mature let-7a-1 (mMIRLET7A1), respectively (Fig. 1b, b).

\section{Exogenous expression of mature miR-223 can effectively inhibit target gene expression in human cells}

It has been well established that miR-223 functions as an oncomiR and regulates numerous target genes in cell proliferation and other cellular functions [34, 69-71]. To test whether the mMIR223 expression vector would produce functional mature miR-223 and regulate some of the target gene expression in a similar fashion to the conventional expression system pMIR223, we transfected these three vectors into two human cell lines, HEK-293 and HCT116. Using qPCR analysis, we found that all three vectors expressed high levels of miR-223 in HEK-293 cells, compared with the vector control group, although the expression level in the priMIR223 group was seemingly higher than that of the pMIR223 and mMIR223 groups (Fig. 2a). We selected seven well-characterized miR-223 target genes, including ARRB1, CHUK, FBXW7, NFIA, PRDM, RHOB, and $S T M N$ [34, 70], and analyzed their expression levels in the transfected HEK-293 cells. Our results indicate that, compared with the vector control group, both pMIR223 and mMIR223 groups exhibited significantly reduced expression levels of the tested seven target genes (Fig. 2a). Moreover, the inhibition of the tested seven target genes mediated by both pMIR223 and mMIR223 vectors was as effective as that of the conventional primary miR-223 expression vector priMIR223 (Fig. 2a).

We conducted the same assays in HCT116 cells. Our results showed that high levels of exogenous miR-223 expression were readily detected in HCT116 cells, although the priMIR223 vector seemingly again mediated the highest level of miR-223 expression (Fig. 2b). While the exact cause(s) of the differences in expression levels remain to be understood, it may reflect the fact that CMV promoter may be much stronger than U6 and/or H1 promoters. Upon examining the expression levels of the selected seven target genes, we found that all three vector transfected groups effectively inhibited the expression of these target genes (Fig. 2b). Similar to the results obtained in HEK-293 cells, pMIR223- and mMIR223-mediated inhibition of the target gene expression was statistically insignificant with that of priMIR223. Collectively, these results suggest that our pMIR223 and mMIR223 expression systems may produce functional miR-223 as effectively as the commonly used primary miR223 system.

\section{Exogenous expression of mature miR-223 specifically inhibits the miR-223 reporter activity in human cells}

To further test the functionality of mMIR 223 vector, we constructed the GLuc-based miR-223 reporter, GLuc- 


\section{A HEK-293 Cells}
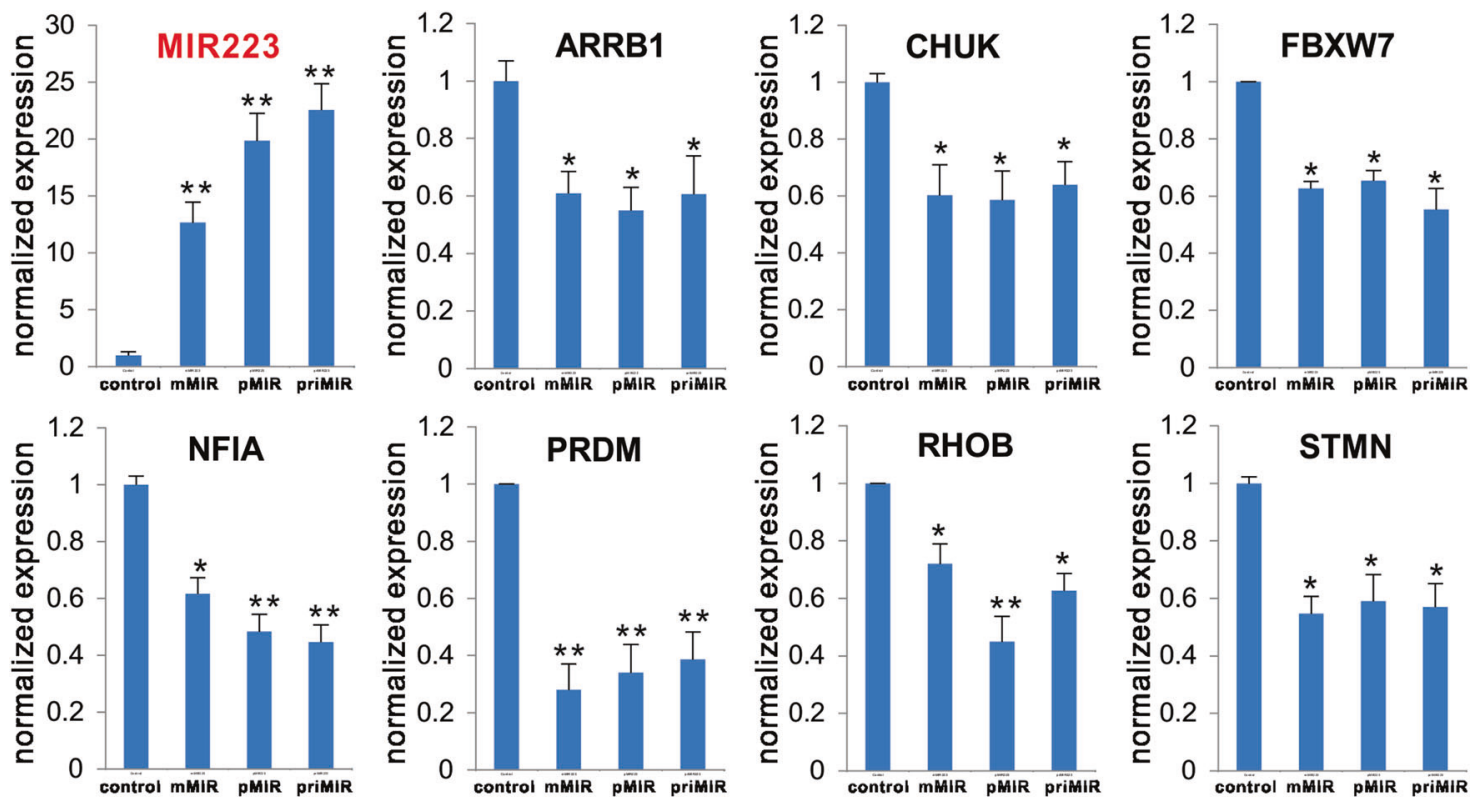

\section{B HCT116 Cells}
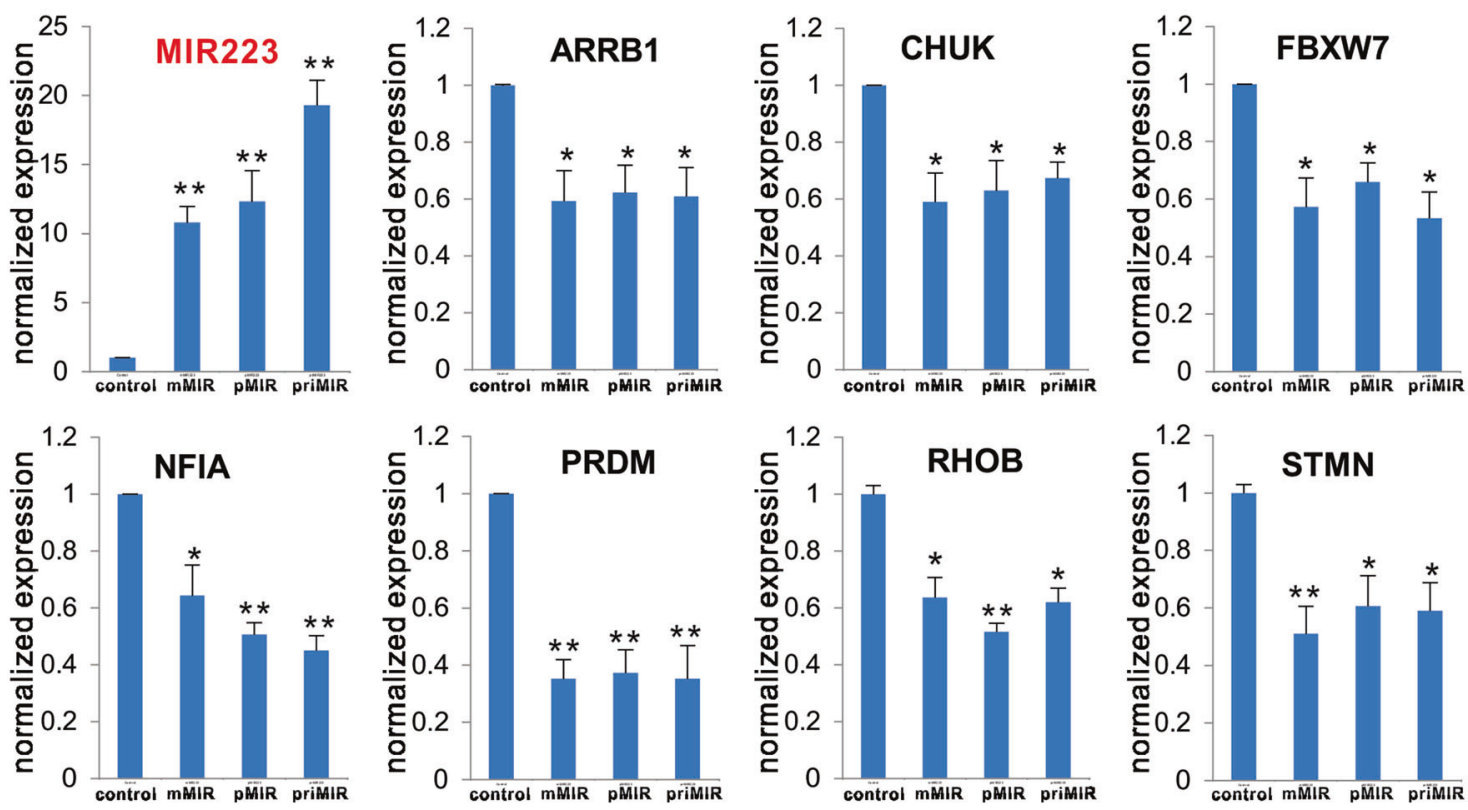

Fig. 2 Comparison of target gene expression inhibition mediated by the three miR-223 expression systems. Confluent HEK-293 (a) and HCT116 (b) cells were transfected with empty vector (Control), mMIR223, pMIR223, or priMIR223. At $72 \mathrm{~h}$ after transfection, total
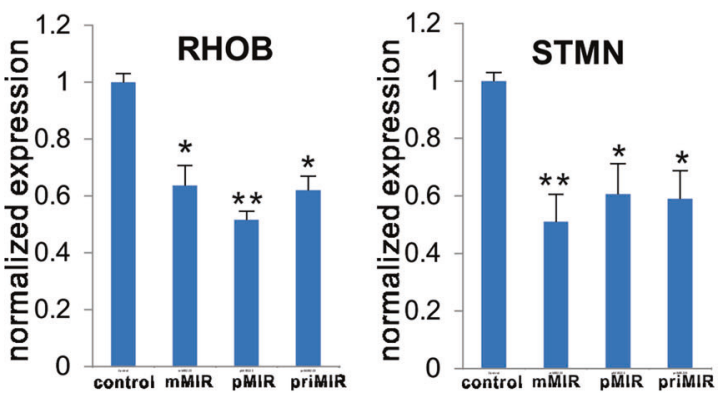

RNA was isolated and subjected to TqPCR analysis. The expression of miR-223 mediated by the three expression systems was also assessed. $* p<0.05$ and $* * p<0.01$ compared with the vector control group

BUTR, by cloning a $200 \mathrm{bp}$ miR-223-binding sitecontaining fragment of the $3^{\prime}$-UTR of human ARRBI transcript (BUTR) into the linker sites of our previously reported miRNA reporter vector pNRGLuc [34]. We also constructed a control reporter by mutating the miR-223binding site of BUTR (i.e., GLuc-BUTR-Mut) (Fig. 3a). When the GLuc-BUTR reporter or the mutant control

reporter GLuc-BUTR Mut was cotransfected with the miR223 expression vectors into HEK-293 cells, we found the all three miR-223 expression vector groups exhibited significantly inhibitory effect on GLuc activities, compared with the mock vector control group (Fig. 3b,a). Furthermore, the inhibitory effect was rather specific as these vectors did not significantly inhibit the GLuc activity of the 


\section{A GLuc-BUTR - hEFH Gluc 3'BUTR-PA- \\ GLuc-BUTR-Mut - hEFH Gluc - Mut -PA-}

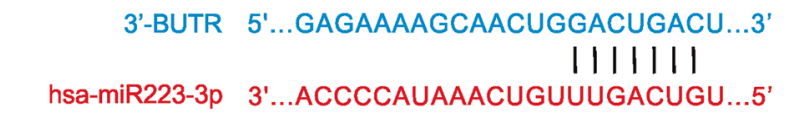

3'-BUTR-Mut 5'...GAGAAAAGCAACUGGUGACUGU....3'
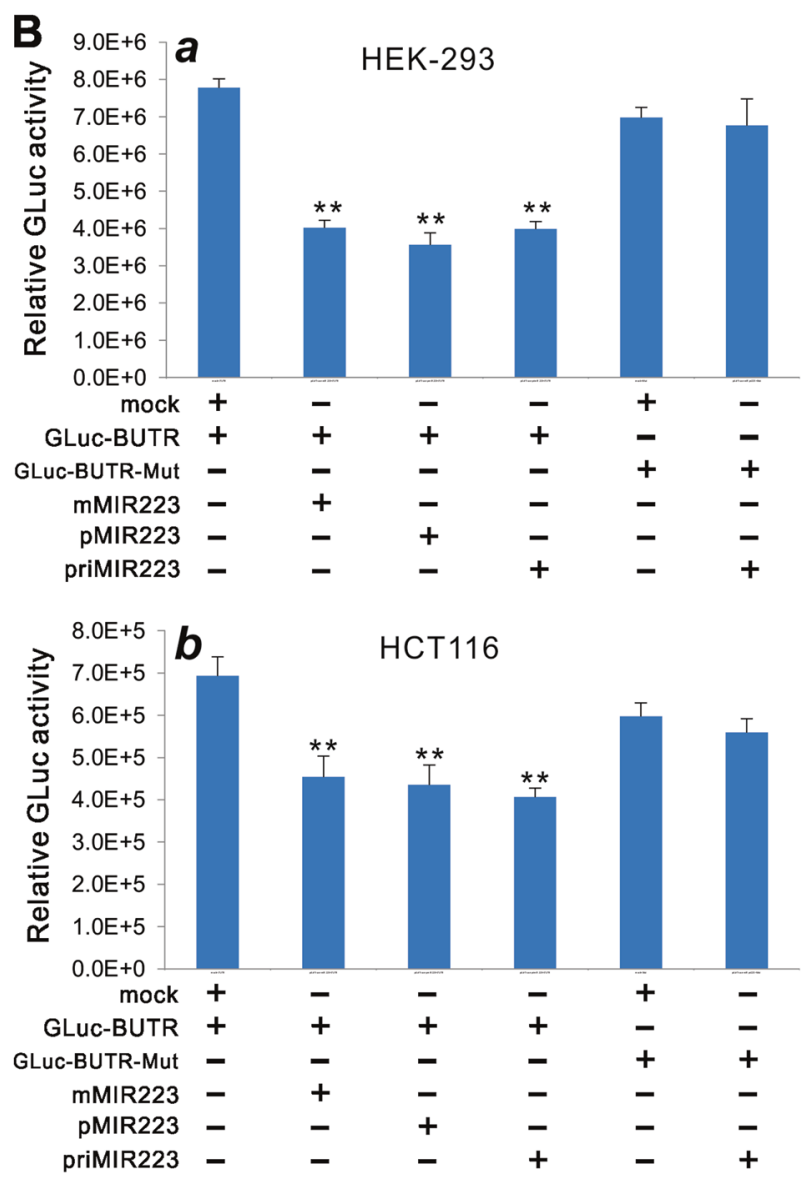

Fig. 3 Comparison of the inhibition of miR-223 reporter activity mediated by the three miR-223 expression systems. a Construction of the miR-223 Gaussia luciferase (GLuc) reporter using the human $\beta$-arrestin $3^{\prime}$-UTR ( $3^{\prime}$ BUTR) that contains a miR-223-binding site. A mutant-binding site reporter (3'BUTR-Mut) was also constructed as a control. b Subconfluent HEK-293 (a) and HCT116 (b) cells were cotransfected with different combinations of reporter vectors and miR223 expression plasmids. GLuc activity was assessed at $72 \mathrm{~h}$ after transfection. Assays were done in triplicate. ${ }^{* *} p<0.01$ compared with the GLuc-BUTR + vector control (mock) group

control reporter, which has the miR-223-binding site mutated (Fig. 3b, a). Similar results were obtained in HCT116 cells. We found all three miR-223 expression vector groups exhibited significantly inhibitory effect on GLuc activities in the GLuc-BUTR group, but not in the GLuc-BUTR-Mut group, compared with the mock vector control group (Fig. 3b, b). These results further suggest that the mMIR223 expression system may produce miR-223 as effectively as the conventional primary miR223 and pMIR223 expression systems.

\section{Exogenous expression of mature miR-223 effectively promotes cell proliferation}

As miR-223 is considered as an oncomiR, we further analyzed the biological effects of the exogenously expressed miR-223 on cell viability and proliferation. When pMIR223 and mMIR223 vectors were transfected in HEK-293 and HCT116 cells, we found that, based on the crystal violet staining assay, cell densities increased significantly, compared with that of the vector control group in both cell lines (Fig. 4a, $a, b$ ). Consistent with the results obtained from the qPCR analysis of the target gene expression and the miR223 reporter assay, the enhancement of cell density in pMIR223 and mMIR223 transfection groups was shown similar to that observed in the priMIR223 (Fig. 4a).

We also carried out the more quantitative WST-1 cell proliferation assay. When the three miR-223 expression vectors, pMIR223, mMIR223, and priMIR223, were transfected in HEK-293 and HCT116 cells, we found that cell proliferation rates were significantly increased at 72 and $96 \mathrm{~h}$ after transfection, compared with that of the vector control group in both cell lines (Fig. 4b, $a, b$ ). Thus, these results collectively demonstrate that the exogenous expression of miR-223 mediated by mMIR223 can achieve similar biological functions conferred by the commonly used priMIR priMIR223 or pMIR223 expression system.

\section{Exogenous expression of mature let-7a-1 (mMIRLET7A1) effectively inhibits target gene expression in human cells}

To ensure the general applicability of the reported pMIRs and mMIR expression vectors, we chose to test another well-studied miRNA let-7a-1 and constructed mature (mMIRLET7A1), as well as the conventional priMIR (priMIRLET7A1) and the precursor (pMIRLET7A1) expression vectors (Fig. $1 \mathrm{~b}, b$ ). We tested the three vectors in two human cell lines, HEK-293 and HCT116, respectively. Using qPCR analysis, we found that all three vectors expressed high levels of let-7a-1 in HEK-293 cells, compared with the vector control group (Fig. 5a). The expression of three let-7a-1 target genes, LIN28, HMGA2, and CMYC [72-74], was effectively inhibited by mMIRLET7A1 at a comparable level with that of priMIRLET7A1 or pMIRLET7A1, compared with that of the vector control group (Fig. 5a).

The same assays were carried out in HCT116 cells. We found that high levels of exogenous let-7a-1 expression were readily detected in HCT116 cells (Fig. 5b). Upon examining the expression levels of the test three target 

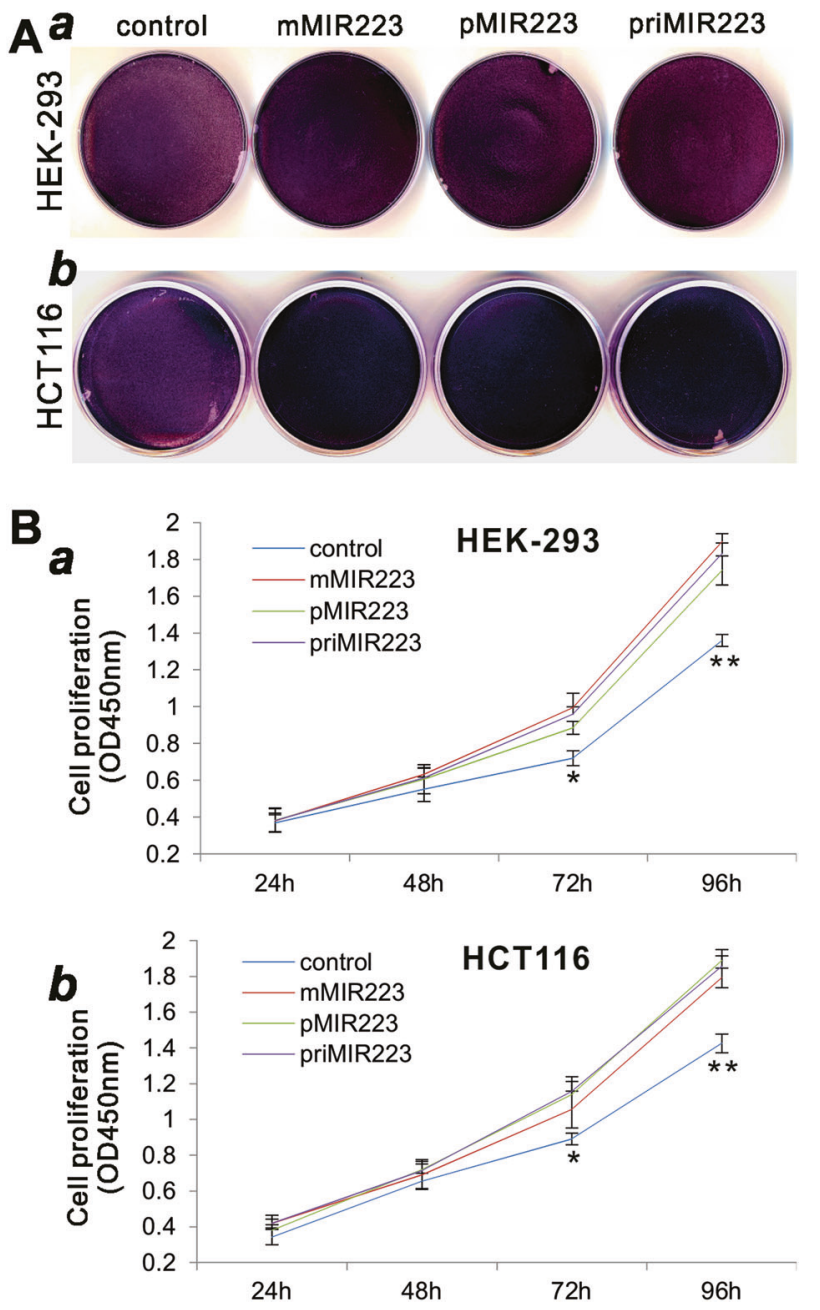

Fig. 4 Comparison of the augmented cell proliferative activity mediated by the three miR-223 expression systems. a The crystal violet assay. Subconfluent HEK-293 (a) and HCT116 (b) cells were transfected with the indicated miR-223 expression plasmids or vector control. Cells were fixed for Crystal violet staining at 3 days after transfection. Assays were done in triplicate, and representative results are shown. b The WST-1 cell proliferation assay. Subconfluent HEK293 (a) and HCT116 (b) cells were transfected with the indicated miR223 expression plasmids or vector control. At the indicated time points, WST-1 substrate was added to each well and incubated for $2 \mathrm{~h}$, followed by measuring the absorbance at $450 \mathrm{~nm}$. Assays were done in triplicate. $* p<0.05$ and $* * p<0.01$ compared with the vector control group

genes, we found that all three vector transfected groups effectively inhibited the expression of these target genes (Fig. 5b). Furthermore, the inhibited expression of the target genes mediated by mMIRLET7A1 was statistically insignificant with that of priMIRLET7A1 or pMIRLET7A1. Thus, these results further suggest that the mMIRLET7A1 expression system may produce functional let-7a-1 as effectively as the conventional primary let-7a-1 or the pMIRLET7A1 expression system.

\section{Exogenous expression of mature let-7a-1 (mMIRLET7A1) specifically inhibits the let-7a-1 reporter activity in human cells}

We further constructed a GLuc-based let-7a-1 reporter, GLuc-LIN28B, by cloning a 525 bp let-7a-1-binding sitecontaining fragment of the $3^{\prime}$-UTR of human LIN28B transcript into the linker sites of pNRGLuc [34]. A control reporter was also constructed by mutating the let-7a-1binding site (i.e., GLuc-LIN28B-Mut) (Fig. 3a). When the GLuc-LIN28B reporter or the mutant control GLucLIN28B-Mut was cotransfected with the let-7a-1 expression vectors into HEK-293 cells, we found all three let-7a-1 expression vectors exhibited significantly inhibitory effect on GLuc activities, compared with the mock vector control group (Fig. 6b, a). Furthermore, the inhibitory effect was shown specific as these vectors did not significantly inhibit the GLuc activity of the mutant control reporter (Fig. 6b,a). Similarly, in the HCT116 cells we found the three let-7a-1 expression vectors exhibited significantly inhibitory effect on GLuc activities of the GLuc-LIN28B reporter, but not in the GLuc-LIN28B-Mut control reporter, compared with the mock vector control group (Fig. $6 \mathrm{~b}, b$ ). These findings further indicate that mMIRLET7A1 expression system can produce functional let-7a-1 as effectively as the conventional primary let-7a-1 or the pMIRLET7A1 expression system.

\section{Exogenous expression of mature let-7a-1 (mMIRLET7A1) effectively inhibits cell proliferation and cell cycle progression}

The let-7a-1 miRNA functions as a tumor-suppressor miRNA [72-75]. Here, we also analyzed the biological effects of the exogenously expressed let-7a-1 on cell viability, cell proliferation, and cell cycle progression. When mMIRLET7A1 vectors were transfected in HEK-293 and HCT116 cells, crystal violet staining assay indicates that the cell densities decreased significantly, similar to that of the priMIRLET7A1 or pMIRLET7A1 transfection group, compared with that of the vector control group in both cell lines (Fig. 7a, $a, b$ ).

The quantitative WST-1 cell proliferation assay also revealed that three let-7a-1 expression vectors, pMIRLET7A1, mMIRLET7A1, and priMIRLET7A1, were transfected in HEK-293 and HCT116 cells, we found that cell proliferation rates were significantly decreased at 72 and $96 \mathrm{~h}$ after transfection, compared with that of the vector control group in both cell lines (Fig. 7b, $a, b$ ). Collectively, these results demonstrate that the exogenous expression of let-7a-1 mediated by mMIRLET7A1 can achieve similar biological functions conferred by the commonly used priMIRLET7A1 or the pMIRLET7A1 expression system.

Lastly, we examined the effect of the exogenously expressed let-7a-1 on cell cycle progression. When 
Fig. 5 Comparison of target gene expression inhibition mediated by the three let-7a-1 expression systems. Confluent HEK-293 (a) and HCT116 (b) cells were transfected with empty vector (control), mMIRLET7A1, pMIR LET7A1, or priMIR LET7A1. At $72 \mathrm{~h}$ after transfection, total RNA was isolated and subjected to TqPCR analysis. The expression of let$7 \mathrm{a}-1$ mediated by the three expression systems was also assessed. $* p<0.05$ and $* * p<$ 0.01 compared with the vector control group

\section{A HEK-293 cells}
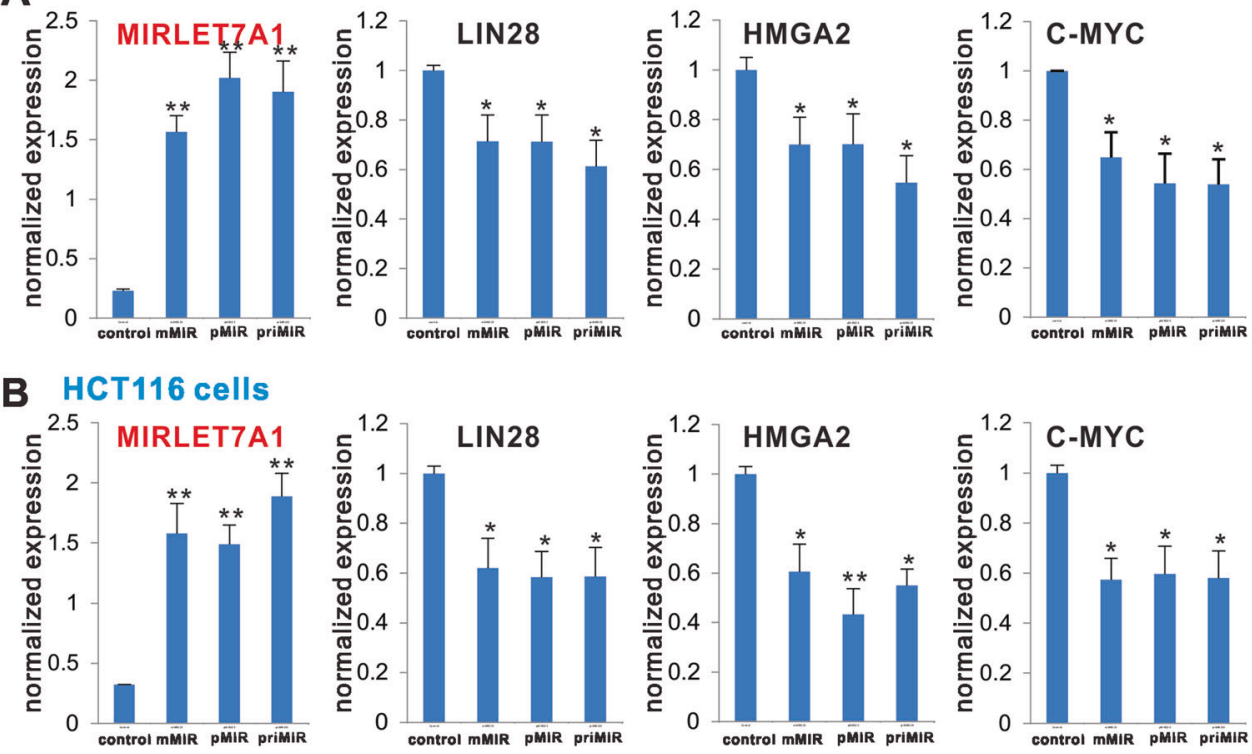

transfected in HEK-293 cells, the mMIRLET7A1 vector, similar to the priMIRLET7A1 or pMIRLET7A1 vector, was shown to increase the $\%$ cells presented in the G1 phase, while decreasing \% cells in the S phase, compared with the vector control (Fig. 7c, $a, b$ ). When transfected into the HCT116 cells, the mMIRLET7A1 vector, along with the priMIRLET7A1 or the pMIRLET7A1 vector, was shown to increase the \% cells presented in the G1 phase (Fig. 7c, $c$, d). Interestingly, unlike in HEK-293 cells, the expression of exogenous let-7a-1 led to decreases of \% cells in the G2/M phases, while no significant changes in the $S$ phase (Fig. 7c, $a b$ vs $c d$ ). Taken together, these results demonstrate that the mMIRLET7A1 vector can express biological active form of let-7a-1 miRNA, which is functionally indistinguishable with that expressed by the conventional primary let-7a-1 miRNA or the pMIRLET7A1 expression system.

\section{Discussion}

In order to develop a user-friendly approach for the effective expression of functional miRNAs, we engineered a simplified strategy to express miRNAs by utilizing the converging $\mathrm{U} 6 / \mathrm{H} 1$ dual promoters to drive the expression of mMIRs. Even though the converging U6/H1 dual promoterdriven system has been successfully used to express siRNAs [39, 40], the asymmetric nature or imperfect complementarity of the $5 \mathrm{p}$-miR and $3 \mathrm{p}$-miR sequences of a given miRNA poses a technical challenge. We overcame this challenge by inserting the transcription stop signals TTTTTAAAAA between the 5p-miR (in sense direction) and $3 p-m i R$ (in antisense direction) sequences to terminate the transcription of $5 \mathrm{p}-\mathrm{miR}$ and $3 \mathrm{p}-\mathrm{miR}$, respectively.
By comparing with the commonly-used priMIR and pMIR expression systems, we demonstrated that the oncomiR hsa-miR-223 expressed in the mature (mMIR223) form effectively inhibited target gene expression, specifically suppressed miR-223 reporter activity, and promoted cell proliferation of HEK-293 and HCT116 cells, in a similar fashion comparable with that of the primary miR-223 or the precursor miR-223 (pMIR223) expression system. Similarly, the tumor-suppressor hsa-let-7a-1 expressed in the mature let-7a-1 forms effectively inhibited the expression of target genes, the reporter activity derived from LIN28B 3'-UTR, and cell proliferation and cell cycle progression, which were indistinguishable from the conventionally expressed primary let-7a-1 or precursor let-7a-1. It is noteworthy that our mMIR expression vectors were constructed on the bases of retroviral transfer and adenoviral shuttle vectors. Thus, the final miRNA expression constructs can be easily converted into recombinant retrovirus for stable expression or recombinant adenovirus for effective transient expression in vitro and in vivo. Taken together, our results demonstrate that the simplified mMIR expression system is user-friendly and reproducibly effective, which should be a valuable resource for studying miRNA functions and/or exploring potential applications of miRNA-based therapeutics.

It has long been a challenge to overexpress functional miRNAs in an effective and reliable fashion. In most cases, overexpression of miRNAs is accomplished by the use of chemically synthesized miRNA mimics, which are mostly made through commercial sources. While this approach is effective and has been widely used, the efficacy of miRNA mimics is transient in nature and relies on transfection efficiency, which may vary drastically in different cell lines. Furthermore, it may be technically challenging and/or 


\section{A GLuc-LIN28B hEFH GLUC 3'UTR PA- GLuc-LIN28B-Mut - hEFH GLuc - Mut PA-}

$\begin{array}{rr}\text { LIN28B 3'-UTR } & \text { 5'...UACUGAUGAUAGUAACUACCUCU...3' } \\ \text { hsa-MIRLET7A-5p } & \text { 3'...UUGAUAUGUUGGAUGAUGGAGU...5' } \\ \text { Mut 3'-UTR } & 5^{\prime} \text {....GCCAACGUUCGAUUUGAUGGAGU...3' }\end{array}$
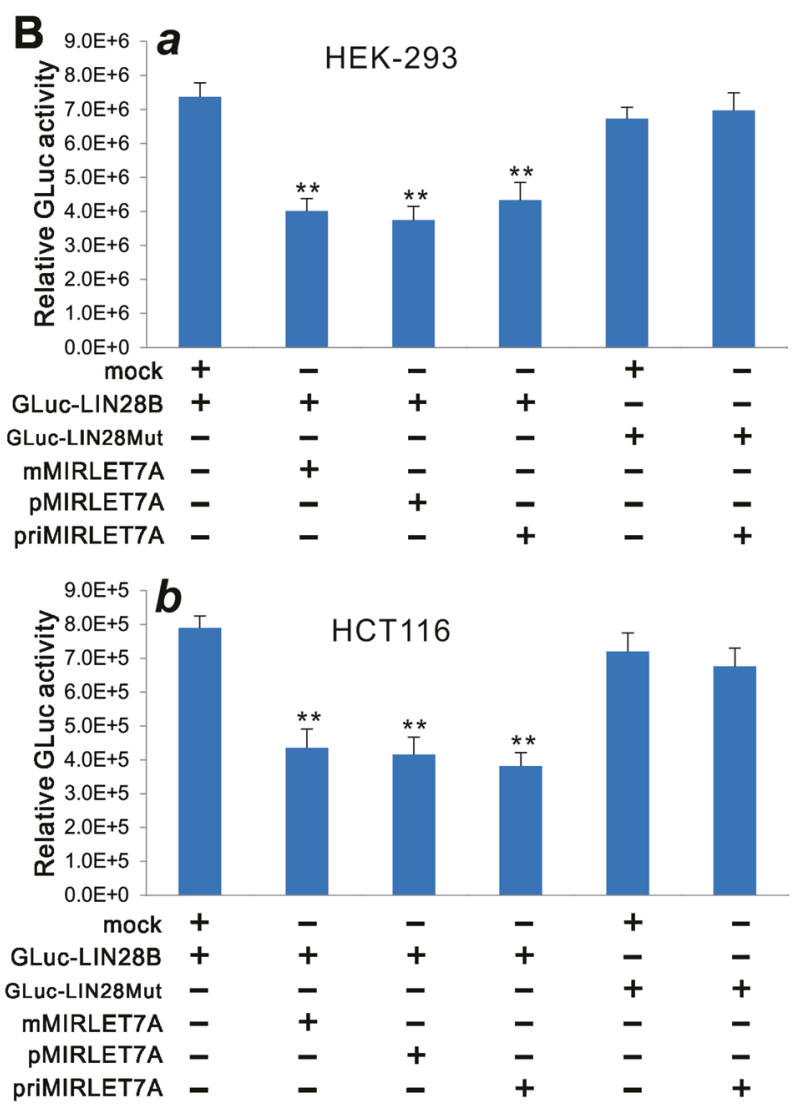

Fig. 6 Comparison of the inhibition of let-7a-1 reporter activity mediated by the three let-7a-1 expression systems. a Construction of the let-7a-1 GLuc reporter using the human LIN28B 3'-UTR that contains a let-7a-1-binding site. A mutant-binding site reporter (Mut 3'-UTR) was also constructed as a control. b Subconfluent HEK-293 (a) and HCT116 (b) cells were cotransfected with different combinations of reporter vectors and let-7a-1 expression plasmids. GLuc activity was assessed at $72 \mathrm{~h}$ after transfection. Assays were done in triplicate. $* * p<0.01$ compared with the GLuc-LIN28B + vector control (mock) group

preventively expensive to use synthesized miRNA mimics for in vivo animal studies.

Several strategies have been used to express miRNAs in the cells and potentially in animals. One commonly employed miRNA expression system is to use a Pol II promoter, such as CMV, SV40, or CMV enhancer/ $\beta$-actin (CA) promoter, or Pol III promoters to drive the expression of a priMIR, which is contained in a $200-500$ bp genomic DNA fragment $[76,77]$. It has been attempted to express multiple miRNAs or shRNA-like stem-loop structures in a single transcript or polycistronic transcript [78-81]. The advantages of this system are the strong transcriptional activity of Pol II promoter and the easy incorporation of induciblity of miRNA expression. In a thorough examination of Pol II- and Pol III-driven expression of shRNA-like stem-loop pri-miRNAs, Furukawa et al. [77] found that, if the construct containing genomic sequences of $100 \mathrm{bp}$ or less flanking the miRNA-encoded region was expressed, the $\mathrm{CMV}, \mathrm{CMVi}$, and CA promoters were more effective than the PGK promoter. However, it is not automatically guaranteed that the priMIRs is effectively processed. In fact, it was shown that miRNA precursors might fail to be efficiently processed to generate the mature forms of miRNAs in some types of cells or under certain conditions [77]. Nonetheless, the U6-driven stem-loop structure of miRNA produced a larger difference between the $5^{\prime}$ - and $3^{\prime}$-strand of the miRNA duplex in miRNA-mediated suppressive effects on reporter gene expression than the pol II promoterderived pri-miRNA [77], suggesting that U6 promoter may be more appropriate for driving the expression of priMIRs. Our results have demonstrated that the pMIR U6-driven primiRNA expression is highly effective and functional.

Another commonly used method takes advantage of the intronic miRNA expression systems [35-37]. This strategy was originated from the discovery of intronic miRNAs (also termed "mirtron"), which are derived from the processing of introns and differ from the intergenic miRNAs in the requirement of RNA polymerase II and splicing machinery components for their biogenesis [82-84]. Such short intronic hairpin miRNAs can bypass Drosha cleavage step, and are instead processed by the splicing machinery and lariatdebranching enzyme to yield pMIR-like hairpins [82-85]. Several variations of such intronic miRNA expression system have been devised over the years. Wu et al. [35, 36] constructed the miRNA and shRNA expression vectors, such as pSM155 and pSM30, by placing miRNA-based artificial miRNA expression cassettes inside of synthetic introns to take advantage of miRNA processing and RNA splicing mechanisms. More recently, a multiplexed miRNA and transgene expression platform exploiting native intronic miRNA loci was reported to accomplish simultaneous repression and expression of protein coding sequences, which may facilitate applications of simultaneous gene silencing and corrective transgene expression in some cases, such as polygenic therapeutic targets, drug resistance genes, viral and host genes involved in the viral life cycle, or oncogenes and tumor suppressor genes [37]. However, the identification of natural human intronic miRNA sequences may be challenging, if not impractical.

A major challenge for exogenously overexpressing priMIR, shRNA-like, or pMIRs is that the production efficiency of mMIRs is dependent on the endogenous miRNA processing and can cause cytotoxicity due to oversaturation 
Fig. 7 Comparison of the let-7a1 -inhibited cell proliferative activity mediated by the three let-7a-1 expression systems. a The crystal violet assay. Subconfluent HEK-293 (a) and HCT116 (b) cells were transfected with the indicated let-7a-1 expression plasmids or vector control. Cells were fixed for Crystal violet staining at 3 days after transfection. Assays were done in triplicate, and representative results are shown. b The WST-1 cell proliferation assay. Subconfluent HEK-293 (a) and HCT116 (b) cells were transfected with the indicated let-7a-1 expression plasmids or vector control. At the indicated time points, WST-1 substrate was added to each well and incubated for $2 \mathrm{~h}$, followed by measuring the absorbance at $450 \mathrm{~nm}$. Assays were done in triplicate. $* p<0.05, * * p<0.01$ compared with the vector control group. c Cell cycle analysis. Subconfluent HEK-293 $(a, b)$ and HCT116 $(c, d)$ cells were transfected with the indicated let-7a-1 expression plasmids or vector control. At $48 \mathrm{~h}$ after transfection, cells were harvested and subjected to cell cycle analysis. Assays were done in triplicate, and representative results are shown $(a, c)$. Quantitative analyses were carried out to determine the $\%$ cell counts in various phases $(b, d) .{ }^{*} p<0.05$ and $* * p<0.01$ compared with the vector control group
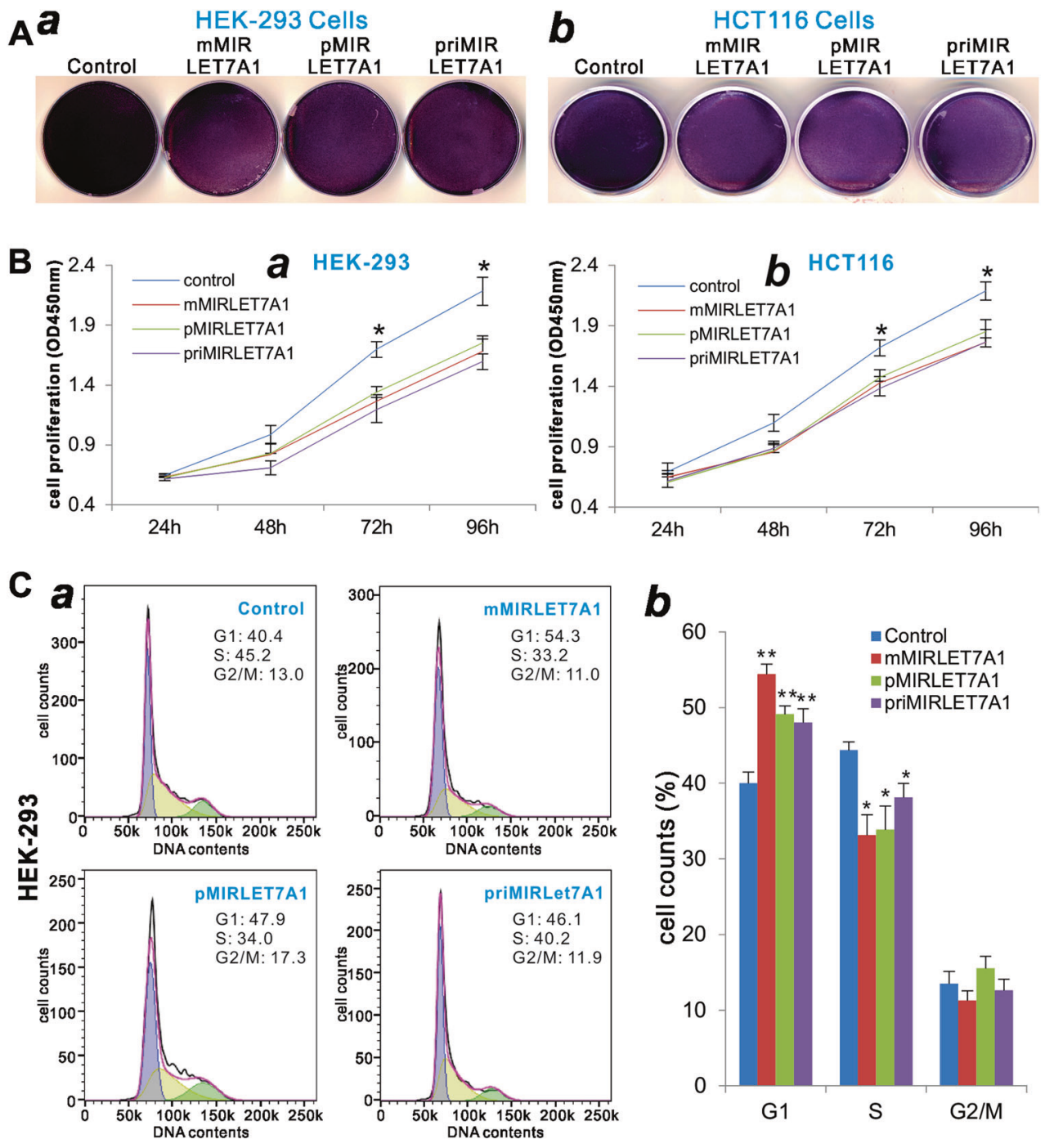

b
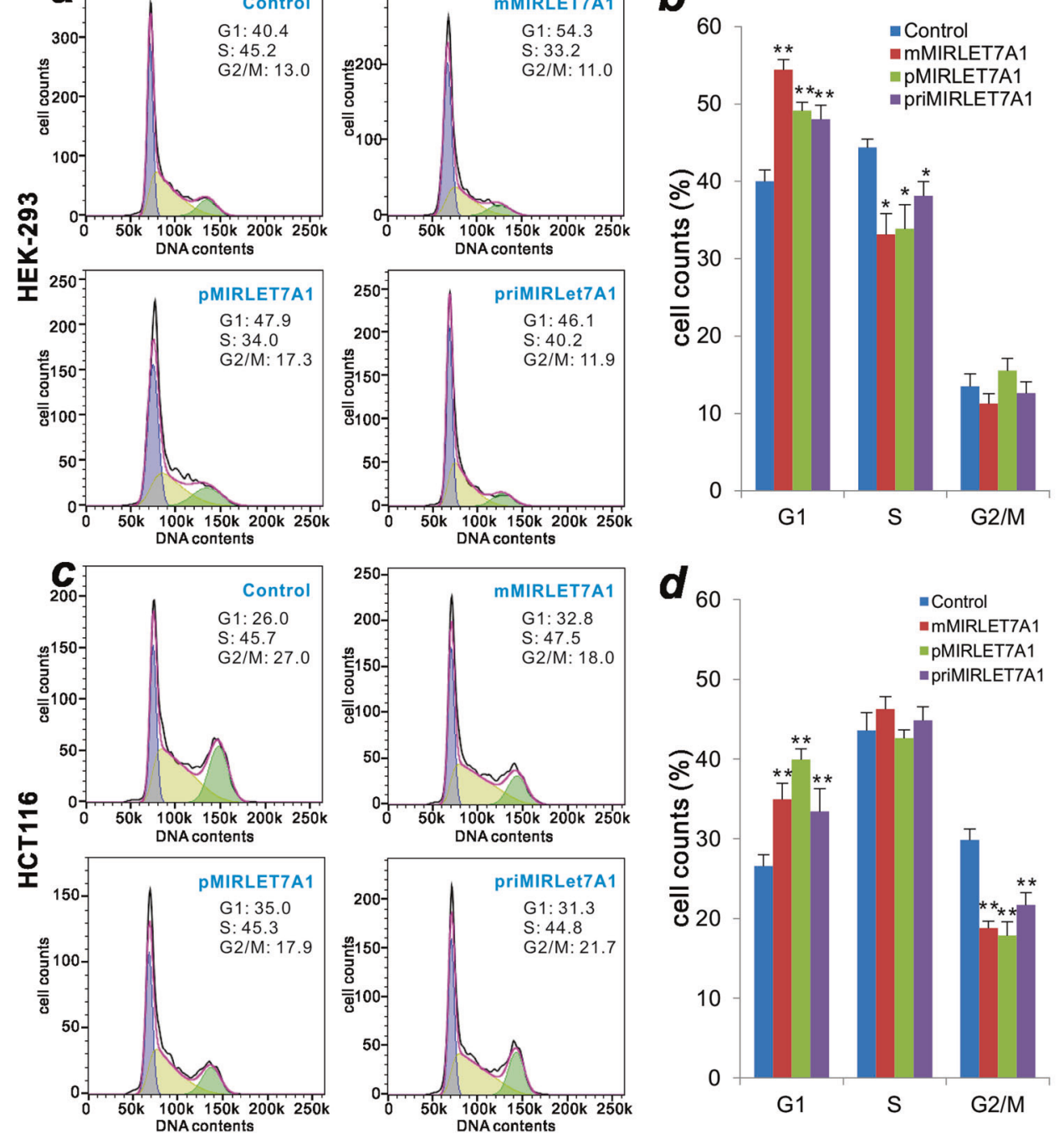

of the RNAi processing machinery [37, 38, 86]. In fact, Ago2 was identified as the primary rate-limiting determinant of both in vitro and in vivo RNAi efficacy, toxicity, and persistence [86]. It was shown that in a mouse study, vector-based Ago-2/Xpo-5 co-expression enhanced U6driven shRNA silencing of exogenous and endogenous 
hepatic targets, reduced hepatotoxicity, and extended RNAi stability by more than 3 months [86]. Ago2 overexpression was shown to significantly boost mRNA silencing efficiency in cell culture by up to tenfold, accompanied with reduced off-targeting effects [87].

Taking together, here we developed a novel and simplified strategy to effectively express mMIRs. This system should theoretically bypass most of the siRNA/miRNA processing machinery, including the involvement of Drosha, Expotin-5, and Dicer1, although it still requires the Ago proteins to fulfill gene silencing function. Therefore, our mMIR expression system is highly customizable and should broadly improve the in vitro and in vivo applications of miRNA experimentations.

Acknowledgements The reported work was supported in part by research grants from the Postdoctoral Research Foundation of China (JF), the Chongqing Postdoctoral Innovation Talent Support Program (JF), the National Key Research and Development Program of China (2016YFC1000803 and 2011CB707906), the National Institutes of Health (CA226303 to TCH), the U.S. Department of Defense (OR130096 to JMW), and the Scoliosis Research Society (TCH and MJL). YF, RZ, ZZ, BH, and BZ were recipients of the predoctorate fellowship from the China Scholarship Council. This project was also supported in part by the University of Chicago Cancer Center Support Grant (P30CA014599) and the National Center for Advancing Translational Sciences of the National Institutes of Health through Grant Number UL1 TR000430. TCH was also supported by the Mabel Green Myers Research Endowment Fund and the University of Chicago Orthopaedic Surgery Alumni Fund. Funding sources were not involved in the study design; in the collection, analysis and interpretation of the data; in the writing of the report; and in the decision to submit the paper for publication.

\section{Compliance with ethical standards}

Conflict of interest The authors declare that they have no conflict of interest.

Publisher's note: Springer Nature remains neutral with regard to jurisdictional claims in published maps and institutional affiliations.

Open Access This article is licensed under a Creative Commons Attribution 4.0 International License, which permits use, sharing, adaptation, distribution and reproduction in any medium or format, as long as you give appropriate credit to the original author(s) and the source, provide a link to the Creative Commons license, and indicate if changes were made. The images or other third party material in this article are included in the article's Creative Commons license, unless indicated otherwise in a credit line to the material. If material is not included in the article's Creative Commons license and your intended use is not permitted by statutory regulation or exceeds the permitted use, you will need to obtain permission directly from the copyright holder. To view a copy of this license, visit http://creativecommons. org/licenses/by/4.0/.

\section{References}

1. Mattick JS, Makunin IV. Non-coding RNA. Hum Mol Genet. 2006;1:R17-29.15 Spec No.
2. Guttman M, Amit I, Garber M, French C, Lin MF, Feldser D, et al. Chromatin signature reveals over a thousand highly conserved large non-coding RNAs in mammals. Nature. 2009;458:223-7.

3. Carninci P, Kasukawa T, Katayama S, Gough J, Frith MC, Maeda $\mathrm{N}$, et al. The transcriptional landscape of the mammalian genome. Science. 2005;309:1559-63.

4. Djebali S, Davis CA, Merkel A, Dobin A, Lassmann T, Mortazavi A, et al. Landscape of transcription in human cells. Nature. 2012;489:101-8.

5. Quinn JJ, Chang HY. Unique features of long non-coding RNA biogenesis and function. Nat Rev Genet. 2016;17:47-62.

6. St Laurent G, Wahlestedt C, Kapranov P. The landscape of long noncoding RNA classification. Trends Genet. 2015;31:239-51.

7. Cech TR, Steitz JA. The noncoding RNA revolution-trashing old rules to forge new ones. Cell. 2014;157:77-94.

8. Sabin LR, Delas MJ, Hannon GJ. Dogma derailed: the many influences of RNA on the genome. Mol Cell. 2013;49:783-94.

9. Brosnan CA, Voinnet O. The long and the short of noncoding RNAs. Curr Opin Cell Biol. 2009;21:416-25.

10. Jacquier A. The complex eukaryotic transcriptome: unexpected pervasive transcription and novel small RNAs. Nat Rev Genet. 2009;10:833-44.

11. Kapranov P, Willingham AT, Gingeras TR. Genome-wide transcription and the implications for genomic organization. Nat Rev Genet. 2007;8:413-23.

12. Soifer HS, Rossi JJ, Saetrom P. MicroRNAs in disease and potential therapeutic applications. Mol Ther. 2007;15:2070-9.

13. Gebert LFR, MacRae IJ. Regulation of microRNA function in animals. Nat Rev Mol Cell Biol. 2019;20:21-37.

14. Murchison EP, Hannon GJ. miRNAs on the move: miRNA biogenesis and the RNAi machinery. Curr Opin Cell Biol. 2004;16:223-9.

15. Chen K, Rajewsky N. The evolution of gene regulation by transcription factors and microRNAs. Nat Rev Genet. 2007;8:93-103.

16. Ha M, Kim VN. Regulation of microRNA biogenesis. Nat Rev Mol Cell Biol. 2014;15:509-24.

17. Treiber T, Treiber N, Meister G. Regulation of microRNA biogenesis and its crosstalk with other cellular pathways. Nat Rev Mol Cell Biol. 2019;20:5-20.

18. Rupaimoole R, Slack FJ. MicroRNA therapeutics: towards a new era for the management of cancer and other diseases. Nat Rev Drug Discov. 2017;16:203-22.

19. Lee RC, Feinbaum RL, Ambros V. The C. elegans heterochronic gene lin-4 encodes small RNAs with antisense complementarity to lin-14. Cell. 1993;75:843-54.

20. Wightman B, Ha I, Ruvkun G. Posttranscriptional regulation of the heterochronic gene lin-14 by lin- 4 mediates temporal pattern formation in C. elegans. Cell. 1993;75:855-62.

21. Lee R, Feinbaum R, Ambros V. A short history of a short RNA. Cell. 2004;116:S89-92. 1 p following S6

22. Kozomara A, Griffiths-Jones S. miRBase: annotating high confidence microRNAs using deep sequencing data. Nucleic Acids Res. 2014;42:D68-73. Database issue

23. Friedman RC, Farh KK, Burge CB, Bartel DP. Most mammalian mRNAs are conserved targets of microRNAs. Genome Res. 2009;19:92-105.

24. Swarts DC, Jore MM, Westra ER, Zhu Y, Janssen JH, Snijders $\mathrm{AP}$, et al. DNA-guided DNA interference by a prokaryotic Argonaute. Nature. 2014;507:258-61.

25. Jonas S, Izaurralde E. Towards a molecular understanding of microRNA-mediated gene silencing. Nat Rev Genet. 2015;16:421-33.

26. Esteller M. Non-coding RNAs in human disease. Nat Rev Genet. 2011;12:861-74

27. Lin S, Gregory RI. MicroRNA biogenesis pathways in cancer. Nat Rev Cancer. 2015;15:321-33. 
28. Bracken CP, Scott HS, Goodall GJ. A network-biology perspective of microRNA function and dysfunction in cancer. Nat Rev Genet. 2016;17:719-32.

29. Ventura A, Young AG, Winslow MM, Lintault L, Meissner A, Erkeland SJ, et al. Targeted deletion reveals essential and overlapping functions of the miR-17 through 92 family of miRNA clusters. Cell. 2008;132:875-86.

30. Takamizawa J, Konishi H, Yanagisawa K, Tomida S, Osada H, Endoh $\mathrm{H}$, et al. Reduced expression of the let-7 microRNAs in human lung cancers in association with shortened postoperative survival. Cancer Res. 2004;64:3753-6.

31. Lu J, Getz G, Miska EA, Alvarez-Saavedra E, Lamb J, Peck D, et al. MicroRNA expression profiles classify human cancers. Nature. 2005;435:834-8.

32. Bak RO, Hollensen AK, Mikkelsen JG. Managing microRNAs with vector-encoded decoy-type inhibitors. Mol Ther. 2013;21:1478-85.

33. Bak RO, Mikkelsen JG. miRNA sponges: soaking up miRNAs for regulation of gene expression. Wiley Inter Rev Rna. 2014;5:317-33.

34. Shu Y, Wu K, Zeng Z, Huang S, Ji X, Yuan C, et al. A simplified system to express circularized inhibitors of miRNA for stable and potent suppression of miRNA functions. Mol Ther Nucleic Acids. 2018;13:556-67.

35. Wu J, Bonsra AN, Du G. pSM155 and pSM30 vectors for miRNA and shRNA expression. Methods Mol Biol. 2009;487:205-19.

36. Wu P, Wilmarth MA, Zhang F, Du G. miRNA and shRNA expression vectors based on mRNA and miRNA processing. Methods Mol Biol. 2013;936:195-207.

37. Seyhan AA. A multiplexed miRNA and transgene expression platform for simultaneous repression and expression of protein coding sequences. Mol Biosyst. 2016;12:295-312.

38. Grimm D, Streetz KL, Jopling CL, Storm TA, Pandey K, Davis CR, et al. Fatality in mice due to oversaturation of cellular microRNA/ short hairpin RNA pathways. Nature. 2006;441:537-41.

39. Luo Q, Kang Q, Song WX, Luu HH, Luo X, An N, et al. Selection and validation of optimal siRNA target sites for RNAi-mediated gene silencing. Gene. 2007;395:160-9.

40. Deng F, Chen X, Liao Z, Yan Z, Wang Z, Deng Y, et al. A simplified and versatile system for the simultaneous expression of multiple siRNAs in mammalian cells using Gibson DNA Assembly. PLoS One. 2014;9:e113064.

41. Shu Y, Yang C, Ji X, Zhang L, Bi Y, Yang K. et al. Reversibly immortalized human umbilical cord-derived mesenchymal stem cells (UC-MSCs) are responsive to BMP9-induced osteogenic and adipogenic differentiation. J Cell Biochem. 2018;119:8872-86.

42. Yan S, Zhang R, Wu K, Cui J, Huang S, Ji X, et al. Characterization of the essential role of bone morphogenetic protein 9 (BMP9) in osteogenic differentiation of mesenchymal stem cells (MSCs) through RNA interference. Genes Dis. 2018;5:172-84.

43. Yu X, Chen L, Wu K, Yan S, Zhang R, Zhao C, et al. Establishment and functional characterization of the reversibly immortalized mouse glomerular podocytes (imPODs). Genes Dis. 2018:5:137-49.

44. Zeng Z, Huang B, Huang S, Zhang R, Yan S, Yu X, et al. The development of a sensitive fluorescent protein-based transcript reporter for high throughput screening of negative modulators of lncRNAs. Genes Dis. 2018;5:62-74.

45. Luo J, Deng ZL, Luo X, Tang N, Song WX, Chen J, et al. A protocol for rapid generation of recombinant adenoviruses using the AdEasy system. Nat Protoc. 2007;2:1236-47.

46. Wang N, Zhang H, Zhang BQ, Liu W, Zhang Z, Qiao M, et al. Adenovirus-mediated efficient gene transfer into cultured threedimensional organoids. PLoS One. 2014;9:e93608.

47. Zhang $\mathrm{H}$, Wang J, Deng F, Huang E, Yan Z, Wang Z, et al. Canonical Wnt signaling acts synergistically on BMP9-induced osteo/odontoblastic differentiation of stem cells of dental apical papilla (SCAPs). Biomaterials. 2015;39:145-54.
48. Untergasser A, Cutcutache I, Koressaar T, Ye J, Faircloth BC, Remm M, et al. Primer3-new capabilities and interfaces. Nucleic Acids Res. 2012;40:e115.

49. Zhang Q, Wang J, Deng F, Yan Z, Xia Y, Wang Z, et al. TqPCR: a touchdown qPCR assay with significantly improved detection sensitivity and amplification efficiency of SYBR green qPCR. PLoS ONE. 2015;10:e0132666.

50. Zhao C, Zeng Z, Qazvini NT, Yu X, Zhang R, Yan S. et al. Thermoresponsive citrate-based graphene oxide scaffold enhances bone regeneration from BMP9-stimulated adipose-derived mesenchymal stem cells. ACS Biomater Sci Eng. 2018;4:2943-55.

51. Fan J, Wei Q, Liao J, Zou Y, Song D, Xiong D, et al. Noncanonical Wnt signaling plays an important role in modulating canonical Wnt-regulated stemness, proliferation and terminal differentiation of hepatic progenitors. Oncotarget. 2017;8:27105-19.

52. Hu X, Li L, Yu X, Zhang R, Yan S, Zeng Z, et al. CRISPR/Cas9mediated reversibly immortalized mouse bone marrow stromal stem cells (BMSCs) retain multipotent features of mesenchymal stem cells (MSCs). Oncotarget. 2017;8:111847-65.

53. Zhao C, Jiang W, Zhou N, Liao J, Yang M, Hu N, et al. Sox9 augments BMP2-induced chondrogenic differentiation by downregulating Smad7 in mesenchymal stem cells (MSCs). Genes Dis. 2017:4:229-39.

54. Liao J, Wei Q, Zou Y, Fan J, Song D, Cui J, et al. Notch signaling augments BMP9-induced bone formation by promoting the osteogenesis-angiogenesis coupling process in mesenchymal stem cells (MSCs). Cell Physiol Biochem. 2017;41:1905-23.

55. Liao J, Yu X, Hu X, Fan J, Wang J, Zhang Z, et al. IncRNA H19 mediates BMP9-induced osteogenic differentiation of mesenchymal stem cells (MSCs) through Notch signaling. Oncotarget. 2017;8:53581-601.

56. Yu X, Liu F, Zeng L, He F, Zhang R, Yan S, et al. Niclosamide exhibits potent anticancer activity and synergizes with sorafenib in human renal cell cancer cells. Cell Physiol Biochem. 2018;47:957-71.

57. Deng Y, Zhang J, Wang Z, Yan Z, Qiao M, Ye J, et al. Antibiotic monensin synergizes with EGFR inhibitors and oxaliplatin to suppress the proliferation of human ovarian cancer cells. Sci Rep. 2015;5:17523.

58. Zhang F, Li Y, Zhang H, Huang E, Gao L, Luo W, et al. Anthelmintic mebendazole enhances cisplatin's effect on suppressing cell proliferation and promotes differentiation of head and neck squamous cell carcinoma (HNSCC). Oncotarget. 2017;8:12968-82.

59. Deng Y, Wang Z, Zhang F, Qiao M, Yan Z, Wei Q, et al. A blockade of IGF signaling sensitizes human ovarian cancer cells to the anthelmintic niclosamide-induced anti-proliferative and anticancer activities. Cell Physiol Biochem. 2016;39:871-88.

60. Gao JL, Lv GY, He BC, Zhang BQ, Zhang H, Wang N, et al. Ginseng saponin metabolite 20(S)-protopanaxadiol inhibits tumor growth by targeting multiple cancer signaling pathways. Oncol Rep. 2013;30:292-8.

61. He BC, Gao JL, Luo X, Luo J, Shen J, Wang L, et al. Ginsenoside $\mathrm{Rg} 3$ inhibits colorectal tumor growth through the down-regulation of Wnt/ss-catenin signaling. Int J Oncol. 2011;38:437-45.

62. He BC, Gao JL, Zhang BQ, Luo Q, Shi Q, Kim SH, et al. Tetrandrine inhibits Wnt/beta-catenin signaling and suppresses tumor growth of human colorectal cancer. Mol Pharm. 2011;79:211-9.

63. Lu S, Wang J, Ye J, Zou Y, Zhu Y, Wei Q, et al. Bone morphogenetic protein 9 (BMP9) induces effective bone formation from reversibly immortalized multipotent adipose-derived (iMAD) mesenchymal stem cells. Am J Transl Res. 2016;8:3710-30.

64. Song D, Zhang F, Reid RR, Ye J, Wei Q, Liao J, et al. BMP9 induces osteogenesis and adipogenesis in the immortalized human cranial suture progenitors from the patent sutures of craniosynostosis patients. J Cell Mol Med. 2017;21:2782-95. 
65. Liao Z, Nan G, Yan Z, Zeng L, Deng Y, Ye J, et al. The anthelmintic drug niclosamide inhibits the proliferative activity of human osteosarcoma cells by targeting multiple signal pathways. Curr Cancer Drug Targets. 2015;15:726-38.

66. Li R, Zhang W, Cui J, Shui W, Yin L, Wang Y, et al. Targeting BMP9-promoted human osteosarcoma growth by inactivation of notch signaling. Curr Cancer Drug Targets. 2014;14:274-85.

67. Li Y, Wagner ER, Yan Z, Wang Z, Luther G, Jiang W, et al. The calcium-binding protein S100A6 accelerates human osteosarcoma growth by promoting cell proliferation and inhibiting osteogenic differentiation. Cell Physiol Biochem. 2015;37:2375-92.

68. Zhou L, An N, Haydon RC, Zhou Q, Cheng H, Peng Y, et al. Tyrosine kinase inhibitor STI-571/Gleevec down-regulates the beta-catenin signaling activity. Cancer Lett. 2003;193:161-70.

69. Esquela-Kerscher A, Slack FJ. Oncomirs - microRNAs with a role in cancer. Nat Rev Cancer. 2006;6:259-69.

70. Haneklaus M, Gerlic M, O'Neill LA, Masters SL. miR-223: infection, inflammation and cancer. J Intern Med. 2013;274:215-26.

71. Gao Y, Lin L, Li T, Yang J, Wei Y. The role of miRNA-223 in cancer: function, diagnosis and therapy. Gene. 2017;616:1-7.

72. Thornton JE, Gregory RI. How does Lin28 let-7 control development and disease? Trends Cell Biol. 2012;22:474-82.

73. Lee H, Han S, Kwon CS, Lee D. Biogenesis and regulation of the let-7 miRNAs and their functional implications. Protein Cell. 2016;7:100-13.

74. Balzeau J, Menezes MR, Cao S, Hagan JP. The LIN28/let-7 pathway in cancer. Front Genet. 2017;8:31.

75. Roush S, Slack FJ. The let-7 family of microRNAs. Trends Cell Biol. 2008;18:505-16.

76. Qiu X, Friedman JM, Liang G. Creating a flexible multiple microRNA expression vector by linking precursor microRNAs. Biochem Biophys Res Commun. 2011;411:276-80.
77. Furukawa N, Sakurai F, Katayama K, Seki N, Kawabata K, Mizuguchi H. Optimization of a microRNA expression vector for function analysis of microRNA. J Control Release. 2011;150:94-101.

78. Zhu X, Santat LA, Chang MS, Liu J, Zavzavadjian JR, Wall EA, et al. A versatile approach to multiple gene RNA interference using microRNA-based short hairpin RNAs. BMC Mol Biol. 2007;8:98.

79. Ely A, Naidoo T, Arbuthnot P. Efficient silencing of gene expression with modular trimeric Pol II expression cassettes comprising microRNA shuttles. Nucleic Acids Res. 2009;37:e91.

80. Snyder LL, Ahmed I, Steel LF. RNA polymerase III can drive polycistronic expression of functional interfering RNAs designed to resemble microRNAs. Nucleic Acids Res. 2009;37:e127.

81. Osorio L, Gijsbers R, Oliveras-Salva M, Michiels A, Debyser Z, Van den Haute $C$, et al. Viral vectors expressing a single microRNA-based short-hairpin RNA result in potent gene silencing in vitro and in vivo. J Biotechnol. 2014;169:71-81.

82. Lin SL, Ying SY. Gene silencing in vitro and in vivo using intronic microRNAs. Methods Mol Biol. 2006;342:295-312.

83. Ruby JG, Jan $\mathrm{CH}$, Bartel DP. Intronic microRNA precursors that bypass Drosha processing. Nature. 2007;448:83-6.

84. Okamura K, Hagen JW, Duan H, Tyler DM, Lai EC. The mirtron pathway generates microRNA-class regulatory RNAs in Drosophila. Cell. 2007;130:89-100.

85. Westholm JO, Lai EC. Mirtrons: microRNA biogenesis via splicing. Biochimie. 2011;93:1897-904.

86. Grimm D, Wang L, Lee JS, Schurmann N, Gu S, Borner K, et al. Argonaute proteins are key determinants of RNAi efficacy, toxicity, and persistence in the adult mouse liver. J Clin Investig. 2010;120:3106-19.

87. Borner K, Niopek D, Cotugno G, Kaldenbach M, Pankert T, Willemsen $\mathrm{J}$, et al. Robust RNAi enhancement via human Argonaute-2 overexpression from plasmids, viral vectors and cell lines. Nucleic Acids Res. 2013;41:e199.

\title{
Affiliations
}

\author{
Jiaming Fan ${ }^{1,2} \cdot$ Yixiao Feng ${ }^{2,3} \cdot$ Ruyi Zhang ${ }^{2,4} \cdot$ Wenwen Zhang ${ }^{2,3} \cdot$ Yi Shu $^{2,3} \cdot$ Zongyue Zeng $^{1,2} \cdot$ Shifeng Huang ${ }^{2,3} \cdot$ \\ Linghuan Zhang ${ }^{2,3} \cdot$ Bo Huang ${ }^{1,2,5} \cdot \mathrm{Di} \mathrm{Wu}^{2} \cdot$ Bo Zhang ${ }^{2,6} \cdot \mathrm{Xi}_{\text {Wang }}{ }^{1,2} \cdot \mathrm{Yan}^{2} \mathrm{Le}^{2,3} \cdot$ Zhenyu Ye $^{2,7} \cdot$ Ling Zhao $^{2,3}$. \\ Daigui $\mathrm{CaO}^{2,3,8} \cdot$ Lijuan Yang ${ }^{2,6} \cdot$ Xian Chen $^{2,9} \cdot$ Bin Liu $^{2,10} \cdot$ William Wagstaff ${ }^{2} \cdot$ Fang He $^{2,3} \cdot$ Xiaoxing $\mathrm{Wu}^{2,3}$. \\ Jing Zhang ${ }^{2,3} \cdot$ Jennifer Moriatis Wolf ${ }^{2} \cdot$ Michael J. Lee $^{2} \cdot$ Rex C. Haydon ${ }^{2} \cdot$ Hue H. Luu $^{2} \cdot$ Ailong Huang $^{1}$. \\ Tong-Chuan $\mathrm{He}^{2} \cdot$ Shujuan $\mathrm{Yan}^{2,11}$
}

1 Ministry of Education Key Laboratory of Diagnostic Medicine, and the School of Laboratory Medicine, Chongqing Medical University, 400016 Chongqing, China

2 Molecular Oncology Laboratory, Department of Orthopaedic Surgery and Rehabilitation Medicine, The University of Chicago Medical Center, Chicago, IL 60637, USA

3 The Affiliated Hospitals of Chongqing Medical University, 400016 Chongqing, China

4 Department of Clinical Laboratory Medicine, The First Affiliated Hospital of Guiyang College of Traditional Chinese Medicine, 550001 Guiyang, China

5 Department of Clinical Laboratory Medicine, The Second Affiliated Hospital of Nanchang University, 330006 Nanchang, China

6 Key Laboratory of Orthopaedic Surgery of Gansu Province, and the Departments of Orthopaedic Surgery and Obstetrics and Gynecology, The First and Second Hospitals of Lanzhou University, 730030 Lanzhou, China

7 Department of General Surgery, The Second Affiliated Hospital of Soochow University, 215004 Suzhou, China

8 Department of Orthopaedic Surgery, Chongqing General Hospital, 400021 Chongqing, China

9 Department of Clinical Laboratory Medicine, The Affiliated Hospital of Qingdao University, 266061 Qingdao, China

10 School of Life Sciences, Southwest University, 400715 Chongqing, China

11 Department of Clinical Laboratory Medicine, Guizhou Provincial People's Hospital and Guizhou University, 550004 Guiyang, China 